Proceedings of the IV Brazilian Conference on Neural Networks - IV Congresso Brasileiro de Redes neurais pp. 400-405, July 20-22, 1999 - ITA, São José dos Campos - SP - Brazil

\title{
Rede Morfológica Não Supervisionada-RMNS
}

\author{
Francisco de Assis Tavares Ferreira da Silva \\ Gerald Jean Francis Banon \\ E-mails: tavares@lac.inpe.br, banon@dpi.inpe.br \\ Instituto Nacional de Pesquisas Espaciais - INPE \\ Av. Dos Astronautas, 1758. Cep: 12.227-010 \\ São José dos Campos - SP, Brasil
}

\begin{abstract}
In this work we propose a new paradigm of Artificial Neural Net (RNA): the Unsupervised Morphologic Net (RMNS portuguese-speaking), characterized by competitive learning. This paradigm belongs to a class of translation invariant nets and it is based on gray level Mathematical Morphology (MM), Carpenter and Grossberg's ART, and Kohonen net models. In the activation phase, the template matching operator is implemented using translation invariant MM elementary operators. These operators enable a robust pattern detection with respect to addictive or subtractive noise, and/or small deformations of the patterns to be recognized. In the training phase, the RMNS uses a Kohonen learning rule variation and a reset system inspired by Carpenter and Grossberger's ART model.
\end{abstract}

\section{Introdução}

As redes morfológicas foram desenvolvidas por pesquisadores interessados na utilização dos operadores morfológicos na área de "Redes Neurais Artificiais" (RNAs), e também por pesquisadores interessados em explorar os mecanismos dos sistemas conexionistas na área da "Morfologia Matemática" (MM) [1, 4, 5, 6, 7 e 8].

No entanto, dentre os trabalhos desenvolvidos, não é explorado e nem sugerido qualquer mecanismo que tire vantagens do formalismo da MM em níveis de cinza [1 e 23], e das características de aprendizagem não supervisionada, dentro de uma abordagem competitiva similar ao modelo de Kohonen [2 e 17], com propriedade de plasticidade e recuperação de padrões (recall do inglês) das redes tipo ART [3].

A rede neural artificial proposta neste trabalho, chamada Rede Morfológica Não Supervisionada (RMNS), é um paradigma de RNA que em tempo de treinamento implementa aprendizagem robusta ao esquecimento, e permite também a geração de protótipos de padrões (classes de padrões representativos) de forma controlada. Em tempo de ativação, a RMNS permite recuperar os protótipos de padrões a partir de padrões semelhantes, isto é, de padrões que estejam dentro de uma faixa de similaridade, estabelecida pelo usuário. Isto corresponde na prática a algum nível de robustez a ruído, variações de forma ou ainda pequenas inclinações (rotações). Em tempo de ativação a rede possui a propriedade de invariância a translação.

A segunda seção apresenta uma introdução à área de Morfologia Matemática, a terceira seção apresenta o paradigma de RMNS proposto. A quarta seção apresenta alguns resultados utilizando o paradigma proposto, e por fim a conclusão e alguns comentários sobre futuros desenvolvimentos.

\section{Introdução à Morfologia Matemática}

A Morfologia Matemática [26 e 27] é a área de processamento de imagens que, baseada na teoria dos reticulados, permite implementar algoritmos utilizados nas tarefas de transformações de padrões em termos dos operadores elementares que são as dilatações, erosões, anti-dilatações e anti-erosões [11, 16, 18 e 19].

Os algoritmos gerados utilizando modelos morfológicos são implementados a partir de equações concisas, expressivas (fáceis de ler e manipular), e que podem gozar de simplificações algébricas. Estas simplificações, permitem, na maioria das vezes, aumentos sensíveis de velocidade de processamento computacional, e/ou redução na quantidade de hardware requerido para a realização da maioria das tarefas de processamento de imagens digitais $[9,10 \mathrm{e}$ 19].

O modelo morfológico para abordagem de problemas de análise de imagem está baseado na extração de informações de imagens a partir de transformações entre reticulados. As transformações singulares crescentes são realizadas através dos operadores elementares, denominados por Matheron [11] e Serra [12] de transformações de dilatação e erosão. Mais tarde, mais duas transformações denominadas de anti-dilatação e anti-erosão, foram introduzidas em [24] que se mostraram últeis para realizar transformações não cresecentes [16 e 18]. No entanto, o formalismo de operadores elementares em 
níveis de cinza, utilizando ELUT (Elementary Look Up Tables), pode ser observado em [1 e 23].

Um reticulado é um conjunto parcialmente ordenado, tal que o ínfimo e o supremo de dois elementos $a$ e $b$ existem [14]. Aqui estes serão respectivamente denotados por $a \wedge b$ e $a \vee b$. Se o reticulado é finito então ele tem um menor e um maior elemento que serão respectivamente denotados como $o$ e $i$. Sejam $\left(L_{1}, \leq\right)$ e $\left(L_{2}, \leq\right)$ dois reticulados finitos e seja $\psi$ um mapeamento de $\left(L_{1}, \leq\right)$ em $\left(L_{2}, \leq\right)$. Aqui $\psi$ será denominado operador. Por definição,

- $\psi$ é uma dilatação $\Leftrightarrow \psi(a \vee b)=\psi(a) \vee \psi(b) e \psi(o)=o$;

- $\psi$ é uma erosão

$\Leftrightarrow \psi(a \wedge b)=\psi(a) \wedge \psi(b) e \psi(i)=i$;

- $\psi$ é uma anti-dilatação $\Leftrightarrow \psi(a \vee b)=\psi(a) \wedge \psi(b) e \psi(o)=i$;

- $\psi$ é uma anti-erosão $\Leftrightarrow \psi(a \wedge b)=\psi(a) \vee \psi(b)$ e $\psi(i)=o$;

para qualquer $a$ e $b$ em $L_{1}$. Os operadores acima são chamados elementares, e tornam possível representar qualquer operador de $\left(L_{1}, \leq\right)$ em $\left(L_{2}, \leq\right)$ [16 e 18].

Introduzimos agora o formalismo usado para representar o casamento de padrões de imagens em níveis de cinza [21 e 25].

Sejam, $\mathbf{Z}$ o conjunto dos números inteiros, $\mathbf{Z}^{\mathbf{2}}$ o produto cartesiano $\mathbf{Z} \times \mathbf{Z}$ (conjunto dos pares ordenados de inteiros), $D \subset \mathbf{Z}^{\mathbf{2}}$ (domínio das imagens), $W \subset \mathbf{Z}^{\mathbf{2}}$ (domínio das imagens ou dos padrões) chamado janela, e $K_{m}$ o intervalo $[0, m] \subset \mathbf{Z}$ (escala de cinza das imagens). Denota-se $K_{m}^{D}$ o conjunto dos mapeamentos de $D$ em $K_{m}$. Estes mapeamentos são representações matemáticas apropriadas para as imagens em níveis de cinza, com domínio $D$ (conjuntos das posições dos pixels) e escala de cinza $K_{m}$, ver Banon [1].

Portanto, as imagens digitais que estão sendo consideradas, podem ser representadas como elementos de $K_{m}^{D}$. Se $f \in K_{m}^{D}$, e $m$ é igual a 1 , então $f$ é uma imagem binária, caso contrário, $f$ é uma imagem em níveis de cinza.

Sejam $f_{W} \in K_{m}^{W}$ (padrão), e $c_{1}, c_{2} \in \mathbf{Z}$ duas constantes tais que $c_{1} \leq c_{2}$.

Denota-se por $f_{W}^{-}$e $f_{W}^{+}$duas imagens em $K_{m}^{W}$, definidas por,

$$
\begin{aligned}
& f_{W}^{-}(x) \stackrel{\Delta}{=} \max \left\{0, \min \left(m, f_{W}(x) \stackrel{\ominus}{+} c_{1}\right)\right\} \\
& f_{W}^{+}(x) \stackrel{\Delta}{=} \max \left\{0, \min \left(m, f_{W}(x)+c_{2}\right)\right\}
\end{aligned}
$$

onde $x \in W$

Sejam $E=D \ominus W$ (onde o símbolo representa a subtração de Minkowski [9]), $l$ um número inteiro em $K_{\mathrm{n}}$ e $i$ um número inteiro no intervalo de 1 a $n$, onde $n$
$=\# W$ (o número de elementos de $W$ ). Denota-se por $\varepsilon_{l}^{i}$ e $\delta_{l}^{a i}$ os operadores de $K_{m}^{D}$ em $K_{1}{ }^{E}$, definidos por

$$
\begin{aligned}
& \mathcal{E}_{l}^{i}(g)(x) \stackrel{\Delta}{=} \begin{cases}1, & \text { se } g\left(x+w_{i}\right) \geq l \\
0, & \text { caso contrário }\end{cases} \\
& \delta_{l}^{a i}(g)(x) \stackrel{\Delta}{=} \begin{cases}1, & \text { se } g\left(x+w_{i}\right) \leq l \\
0, & \text { caso contrário }\end{cases}
\end{aligned}
$$

para todo $g \in K_{m}^{D}$ e $x \in E$.

Seja $i \mapsto w_{i}$ uma bijeção entre $\{1, \ldots, n\}$ e $W$ (enumeração das posições de $W$ ).

Os operadores $\mathcal{E}_{l}^{i}$ são erosões e os $\boldsymbol{\delta}_{l}^{a i}$ são antidilatações de $K_{m}^{D}$ em $K_{1}^{E}$.

Denota-se por $\lambda^{i}$ o operador de $K_{m}^{D}$ em $K_{1}^{E}$, definido por:

$$
\lambda \stackrel{i \Delta}{=} \mathcal{E}_{f_{W(w i)}^{-}}^{i} \wedge \delta_{f_{W(w i)}^{+}}^{a i}
$$

Os operadores $\lambda^{i}$ são operadores supgeradores da morfologia [18].

O operador de $K_{m}^{D}$ em $K_{n}^{E}$ denotado por $\phi$ e denominado por operador de casamento de padrões [21 e 25], é o operador dado por:

$$
\phi \stackrel{\Delta}{=} \sum_{i=1, \ldots, n} \lambda^{i},
$$

O valor $\phi(g)(x)$ mede a similaridade entre o padrão $f_{W}$ e a subimagem de $g$ vista através de $W$ transladado de $x$. No caso da subimagem ser exatamente $f_{W}$, a similaridade será igual a $n$. Se $c_{1} \neq c_{2}$ então o modelo acima permite fazer um casamento com folga nos níveis de cinza.

No final desta seção é apresentado o operador sobre imagens digitais que localiza padrões com valores de similaridade acima ou igual a um determinado limiar em uma imagem digital [22].

Seja $l \in K_{\mathrm{n}}$, o operador de limiarização $\psi_{l}^{*}$ de $K_{n}^{E}$ em $K_{1}$ que localiza os valores de similaridade acima ou igual a $l$ é o operador dado por:

$$
\psi_{l}^{*}(f) \stackrel{\Delta}{=}\left\{\begin{array}{ll}
1, & \text { se } \exists x \in E, f(x) \geq l \\
0, & \text { caso contrário }
\end{array} .\right.
$$

Este operador que é uma dilatação, será muito útil para implementação de operadores de detecção com folga, o qual será tratado a seguir. Para efeitos práticos deve-se compor o operador de limiarização (Expressão 2.5) com o operador de casamento de padrões (Expressão 2.4), esta composição permite a detecção com folga (limiarizada) de padrões [22].

$$
\psi_{l}^{\circ} \circ \phi
$$


onde o símbolo $\circ$ denota a composição de mapeamentos.

\section{Rede morfológica não supervisionada}

A Rede Morfológica Não Supervisionada "RMNS", observada na Figura 1.0, combina uma camada de entrada com uma camada competitiva, mais duas camadas para recuperação e saída de informação, e é treinada por aprendizado não supervisionado. A seguir é apresentado o formalismo do modelo de RNA proposto.

Sejam as funções $f \in K_{m}{ }^{W}, g \in K_{m}{ }^{D}$, e $f_{W} \in K_{m}{ }^{W}$, respectivamente, o padrão a ser treinado, a imagem a ser processada, em tempo de ativação e o tipo dos "pesos" dos neurônios morfológicos. Então pode-se definir uma rede neural morfológica com quatro camadas como a seguir.

A primeira camada, camada de entrada, contém $W$ ou $D$ nós do tipo fan-out. Utiliza-se $W$, caso o usuário não pretenda explorar a capacidade de invariância a translação em tempo de ativação. No entanto, caso o usuário pretenda utilizar a característica de invariância a translação, é importante que seja dimensionado um $D$ que comporte a maior imagem a ser utilizada em tempo de ativação.

A segunda camada, também chamada de mapa morfológico, é uma camada morfológica competitiva constituída por $N$ nós morfológicos tipo $\psi_{l}^{*} \circ \phi$. Denota-se por $z_{j}$ o valor de $\left(\psi_{l}^{\bullet} \circ \phi^{j}\right)(g)$ (saída do nó $j$ ) onde $j=1, \ldots, N$. Os pesos dos neurônios morfológicos são compostos por $f_{w_{j}}^{+}\left(w_{i}\right)$ e $f_{w_{j}}^{-}\left(w_{i}\right) \in K_{m} \operatorname{com} i=1$, ..., $n$.

Para simplificação, defini-se $w_{j i}=f_{w_{j}}^{+}\left(w_{i}\right)=f_{w_{j}}^{-}\left(w_{i}\right)$, pois isso simplificará o número de parâmetros a serem passados a rede.

Os $N$ neurônios da primeira camada interna possuem também duas sinapses inibidoras com pesos especiais $w_{a}^{c}$ e $w_{p}^{c}\left(w_{a}^{c}=1\right.$ e $\left.w_{p}^{c}=1\right)$, responsáveis pela resolução de possíveis conflitos, em tempo de competição realizada nesta camada. Estes neurônios são dotados também de um parâmetro chamado limiar de ativação $w_{j 0}=l$, onde $l \in K_{n}$ (o limiar de ativação é o percentual de aceitação do quanto o usuário deseja aceitar como folga para detecção).

A segunda camada interna, camada de recuperação, com $N$ nós semelhantes ao tipo out-star [15], cuja função de ativação é obtida por uma dilatação de $K_{1}$ em $K_{m}{ }^{W}$

$$
\delta_{w}^{*}(z)=\left\{\begin{array}{ll}
f_{w} & \text { se } z=1 \\
0_{w} & \text { caso contrário }
\end{array},\right.
$$

onde $z$ é a saída do neurônio ativo da primeira camada interna e $0_{W}$ é a imagem preta de domínio $W$.

Denota-se por $s_{j}$ o valor $\delta_{w_{j}}^{*}\left(z_{j}\right)$. A dilatação $\delta_{W}^{*}$

é utilizada como um recurso de recuperação de pesos, semelhante a função de ativação tipo out-star, e o objetivo é recuperar o padrão protótipo, previamente "memorizado" nas sinapses do neurônio em tempo de treinamento.

A última camada, camada de saída, contém um neurônio com pesos unitários, o qual implementa a união de todos os sinais gerados por todos os neurônios da camada anterior. Denotando por y o valor de saída da

rede, a função de ativação para o nó da camada de saída é dada por: $y=\bigcup_{j=1}^{N} s_{j}$.

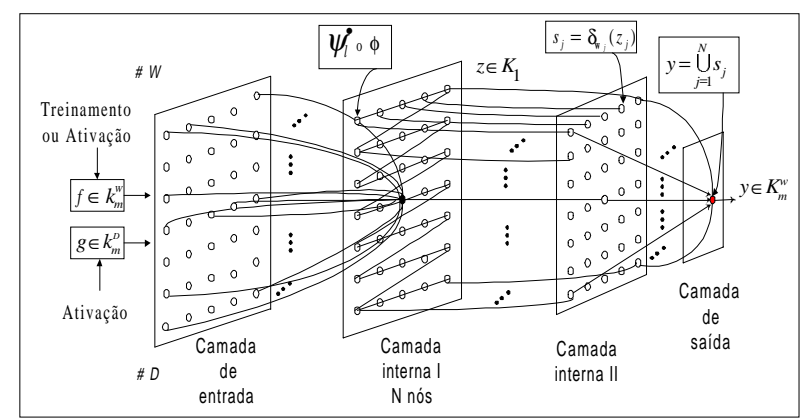

Figura 1.0 Exemplo de RMNS.

\subsection{Princípios de funcionamento da RMNS}

O primeiro passo na operação de treinamento de uma RMNS é computar a detecção com folga, para cada unidade da camada morfológica, isto é, de posse do valor apresentado, via a camada de entrada, os neurônios da camada morfológica são ativados com um operador de detecção com folga (Expressão 2.6). Esse processo verifica se já existe algum neurônio que possa representar o padrão apresentado na camada de entrada, com uma folga dada pelo limiar $l$.

O parâmetro de limiar $l$ pode ser tratado como uma taxa de generalização e é estabelecida de acordo com o percentual de similaridade desejada para um determinado "agrupamento" (classe) de padrões.

Caso não ocorra qualquer ativação com valor maior que zero, uma competição é estabelecida para encontrar uma única unidade vencedora. A competição é implementada através de "sinapses" especiais que permitem verificar qual o neurônio de maior índice apresenta o maior nível de excitação. Este mecanismo é detalhado na Expressão 3.3. 
No caso da necessidade de treinamento, a competição é baseada na distância Euclidiana entre $f$ e $f_{W}$. A unidade que obtém o valor de maior similaridade ganha a competição. Aqui denotamos esta unidade vencedora como $z_{j}^{*}$. A unidade que apresentar menor diferença inibe todas as outras unidades $j$ na camada competitiva. Um modelo de resolução de conflitos de competição pode ser implementado utilizando-se o seguinte mecanismo. Seja a função de avaliação:

$$
z_{j}=F\left(d_{j}\right) * \alpha_{j}
$$

onde:

$$
F\left(d_{j}\right)=\left\{\begin{array}{ll}
1 & \text { se } \min \left(d_{h}\right)>d_{j}(\forall h<j) \text { e } \min \left(d_{k}\right) \geq d_{j}(\forall k>j) \\
0 & c c
\end{array},,\right.
$$

$j>1, d_{j}$ é a distância (ex.: distância Euclidiana) entre os pesos do neurônio e o padrão amostrado, $\min \left(d_{h}\right)$ é a distância mínima dos neurônios anteriores, $\min \left(d_{k}\right)$ é a distância mínima dos neurônios posteriores, $\alpha_{j}=1$ (reset $=1$ ) indica que o neurônio está apto ao treinamento.

\subsection{Auto organização na RMNS}

O modelo de auto-organização que emerge na RMNS é gerado em tempo de aprendizagem, isto é, durante o treinamento a RMNS encontra e ajusta as relações de similaridades entre os padrões e os pesos de cada neurônio. Padrões apresentados são classificados pelas unidades que eles ativam na camada morfológica. As similaridades entre padrões são obtidas por um limiar $l$, pré estabelecido no início do treinamento.

A eliminação de todas as hipóteses de similaridade (pré-ativação) e a determinação do neurônio vencedor é a chave do treinamento da rede. Ao contrário da maioria das redes neurais, na RMNS proposta, somente o neurônio vencedor modifica os pesos em suas conexões, caso nenhum dos neurônios já treinados possam representar o padrão sob treinamento. A modificação dos pesos do neurônio morfológico é baseada no modelo de Kohonen [2 e 17] e é dada por:

$$
w_{j i}^{(\text {novo })}=w_{j i}^{(\text {velho })}+\alpha_{j}\left(x_{i}-w_{j i}^{(\text {velho })}\right) z_{j}^{*}
$$

onde $\alpha_{j}$ é o parâmetro de reset, $x_{i}$ são as componentes do vetor sinal de entrada correspondentes a cada padrão apresentado $\boldsymbol{x} \in K_{m}{ }^{W}, w_{j i} \in K_{m}$, e $z_{j}{ }^{*} \in K_{1}\left(z_{j}{ }^{*}\right.$ é a saída do neurônio vencedor após a competição), para $j=1, \ldots, N$.

Nesta primeira versão de RMNS, a regra (3.4) é equivalente a

No entanto é importante observar que a regra de aprendizagem morfológica é apenas uma variação da

$$
w_{j i}^{\text {novo }}=\left\{\begin{array}{l}
x_{i} \text { se } \alpha_{j}=1 e z_{j}^{*}=1 \\
w_{j i}^{\text {velho }} \quad c c
\end{array}\right.
$$

regra utilizada na Rede de Kohonen e também do sistema de reset da Rede ART. De fato, pode-se observar que tomando-se a vizinhança igual a zero, e considerando-se o fato de que cada neurônio tem seu $\alpha$ (reset) independente e após o treinamento $\alpha_{j}=0$, a regra de aprendizagem morfológica torna-se um caso particular do modelo de aprendizagem de Kohonen,.

Esta regra de aprendizagem considera a coleção de pesos para um certo neurônio como um vetor de peso $N$-dimensional e o correspondente sinal de entrada como um vetor de entrada $\mathrm{N}$-dimensional. Portanto, a regra de aprendizagem morfológica modifica os "vetores peso" de forma que estes se alinhem ao vetor de entrada.

É importante observar que apenas o neurônio vencedor " $z_{j}^{*}$ " tem seus pesos atualizados. O processo de treinamento tenta aglomerar os padrões no mapa morfológico para refletir a variação dos tipos de classes encontrados nos dados de treinamento. Essas são mudanças em grande escala para a orientação dos neurônios no mapa. Portanto, em tempo de treinamento, taxas grandes de generalização deverá permitir agrupamentos com grandes folgas (na maioria das vezes grandes classes com padrões homogêneos), estabelecendo-se assim, um mapa morfológico, o mais rápido possível.

Cada vez que é aplicado uma nova entrada de treinamento não similar a qualquer outra já apresentada à rede, o neurônio vencedor tem que primeiro ser localizado; isso identifica o neurônio do mapa morfológico que terá o seu valor de peso atualizado. $\mathrm{O}$ neurônio vencedor é categorizado como o neurônio que tem o vetor de pesos com maior semelhança (mais próximo em termos de distância Euclidiana) ao vetor de entrada.

O algoritmo de treinamento produzirá representantes para todos os tipos de classes encontradas nos dados de treinamento, caso haja neurônios (memória) suficiente.

Uma vez que a rede implementa auto-organização (seleciona e treina o neurônio mais próximo ao padrão sob treinamento), as representações internas das aglomerações no mapa morfológico podem ser catalogadas para indicar a sua classe de modo que a rede possa ser usada para classificar entradas não conhecidas. Isto é importante quando apenas o mapa morfológico é utilizado, no lugar de uma rede completa. Note que a rede forma as características internas sem supervisão.

Deste ponto de vista, o neurônio vencedor é aquele com vetor peso mais próximo do vetor de entrada, e o resultado do ciclo de treinamento é a "aproximação" do vetor peso que representa o vetor de entrada, isto é, a modificação dos pesos para que representem o padrão de entrada e que possam ser utilizados por operadores de detecção. As regras básicas do mapa morfológico de características podem ser descritas resumidamente como a seguir: 
Inicialmente, deve-se localizar a unidade na camada morfológica cujos pesos têm similaridade suficiente para gerar respostas de ativação para o padrão apresentado. Tenta-se, localizar o neurônio que represente o padrão sob treinamento. Caso algum neurônio já treinado esteja dentro do limiar pré estabelecido, este neurônio é o representante do padrão corrente. Caso ainda não exista representante desta classe, uma competição é estabelecida. O neurônio vencedor informa disponibilidade para aprendizagem. Aumenta-se a similaridade, modificando-se os pesos da unidade vencedora. Em tempo de ativação, a imagem $g$ é apresentada a camada de entrada e é processada através dos operadores de cada camada, conforme Figura 1.0.

\section{Alguns resultados obtidos}

Alguns experimentos já realizados podem mostrar como a RMNS deve contribuir para o desenvolvimento de novos modelos morfológicos adaptativos. A resposta da RMNS apresentada, mostra que a partir de imagens treinadas, contendo vários padrões, a rede contendo uma camada de entrada com 196 neurônios, 36 neurônios na primeira e segunda camadas internas, e um neurônio na camada de saída, foi capaz de identificar os padrões "alfabeto" (com letras contendo $14 \times 14$ pixels) "previamente aprendidos".

A Figura 4.1 mostra os pesos, no mapa morfológico, inicializados aleatoriamente. Enquanto que a Figura 4.2 mostra o mapa após 26 ciclos de treinamento.

\section{Figura 4.1}

Figura 4.2

A Figura 4.3 apresenta pares de "entrada e saída" em tempo de ativação na RMNS, utilizada para recuperação de padrões individuais. Neste primeiro exemplo, foi utilizado um conjunto de treinamento contendo caracteres " $14 \times 14$ pixels" em imagens binárias. Para ativação, foi utilizado o conjunto de padrões submetidos a ruído Gaussiano de $14 \%$ para verificação da robustez de detecção de padrões imperfeitos, e portanto demonstrar a capacidade de generalização do paradigma proposto.

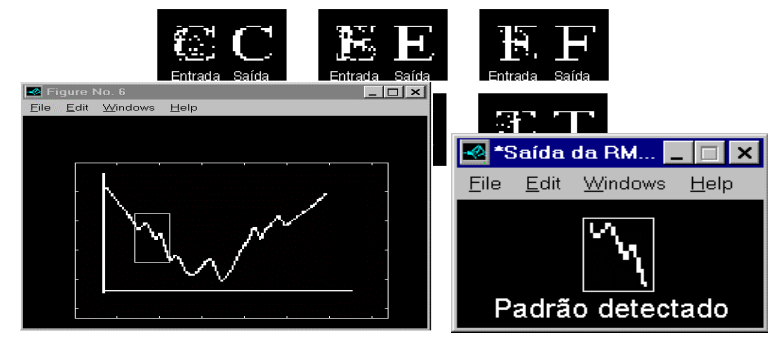

Figura 4.3

Nas Figuras 4.4 e 4.5, pode-se observar a detecção de padrões invariante a translação em um gráfico hipotético relativo a bolsa de valores. Neste exemplo a RMNS detecta uma instabilidade anterior a um período de baixa.

Figura 4.4

Figura 4.5
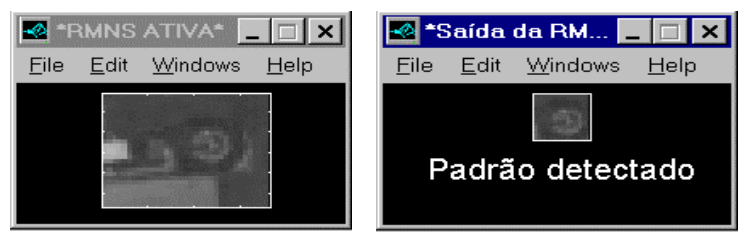

Nas Figuras 4.6 e 4.7, pode-se observar a detecção de um padrão, previamente treinado, em uma imagem em níveis de cinza, relativo a uma janela de uma foto aérea da cidade de São José dos Campos. Neste exemplo a RMNS, treinada com padrões em níveis de cinza, detecta um padrão "alvo" $15 \times 15$ (Figura 4.7) referente a imagem $36 \times 28$ (Figura 5.6) contendo a rotatória próximo ao aeroporto.

Figura 4.6

Figura 4.7

\section{Conclusão}

A utilização de operadores morfológicos na área de redes neurais é promissora tanto em relação as questões de otimização de processamento de informação, como também com referência à área da Morfologia Matemática, o que deverá permitir, por exemplo, a obtenção de um modelo bem conhecido extraído da rede já treinada. Portanto, todas as vantagens de possíveis otimizações algébricas já utilizadas na própria área de MM [1, 10, 19 e 20], podem ser também aproveitadas na área de RNAs. Por outro lado, a MM pode dispor das vantagens dos mecanismos adaptativos nos quais a RMNS está baseada.

Do ponto de vista de pesquisas em novos paradigmas de aprendizagem, a possibilidade de representação e detecção de símbolos utilizando um modelo formal, baseado em MM com capacidade adaptativa, deverá permitir também a análise formal de vários mecanismos relevantes encontrados nas tarefas de interpretação de sinais.

Uma variação importante do mecanismo de treinamento, seria a implementação de sistema de reset dissociado da taxa de aprendizagem. Isto é, uma variação do modelo de RMNS, pode ser construído inserindo-se um parâmetro de reset extra como utilizado pela Rede ART, e utilizando-se a taxa de aprendizagem no seu sentido original como implementado na Rede de Kohonen. Este mecanismo deverá propiciar as vantagens da plasticidade das Redes tipo ART e também explorar a geração de mapas topológicos encontrados nas Redes tipo Kohonen. Outras variações da RMNS para detecção invariante a 
rotação e escala, dentro de um modelo para detecção de mútiplos padrões estão sendo investigadas.

\section{Referências}

[1] Banon, G. J. F. Characterization of translation invariant elementary morphological operators between gray-level images. Relatório de pesquisa INPE-5616-RPQ/671, INPE, São José dos Campos-SP-Brasil, Dezembro 1995. Repositório da URLib: <dpi.inpe.br/banon/1995/10.20.17.45>

[2] Kohonen, T. Self-organized formation of topologically correct feature maps. Biological Cybernetics., 43, 1982.

[3] Carpenter, G. A.; Grossberg, S. ART2: Self organization of stable category recognition codes for analog input patterns. Applied Optics, 26:4919-4930, 1987.

[4] Pessoa, F.C.; Maragos, P. Morphological/Rank neural networks and their adaptive optimal design for image processing, IEEE Conference on Acoustics, Speech \& Signal Processing, 1996.

[5] Brugge, R. J.; Krol R. J.; Nijhuis, J. A. G.; Spaanenburg L. Morphological expressions for DTCNN functions, IEEE-Intenational Conference on Neural Networks, 1996, p. 745-750.

[6] Davidson, J. L.; Hummer, F. Morphology neural networks: An introduction with applications. Circuits System Signal Process, (2), 1993, p. 179-210.

[7] Wilson, S. S. Unsupervised training of structuring elements, SPIE Proceeding in Image Algebra and Morphological Image Processing II, vol. 1568, p. 188199, 1991

[8] Wilson, S. S. Training structuring elements in morphological networks. Mathematical Morphology in Image Processing. Marcel Dekker, Inc., New York, 1993. p. 1-41.

[9] Banon, G. J. F.; Barrera J. Bases da morfologia matemática para análise de imagens binárias. Segunda edição. MCT/INPE, número INPE-6779-RPQ/682, 1998. Repositório da URLib: <dpi.inpe.br/banon/1998/06.30.17.56>.

[10] Haralick, R. M.; Stemberg, S. R.; Zhuang, X. Image analysis using mathematical morphology, IEEE Trans. Pattern Anal. Machine Intell, PAMI-9, p. 532-550, July, 1987.

[11] Matheron, G. Random sets and integrad geometry. New York: Wiley, 1975.

[12] Serra, J. Stereology and structuring elements. J. Microscopy, 1972. p. 93-103.

[13] Matheron, G. Eléments pour une Théorie des Milieux Poreux. Paris: Ed. Masson, 1967.

[14] Birkhoff, G. Lattice theory. 3.ed. Providence, Rhode Island, American Mathematical Society, 1967.

[15] Grossberg, S. Embedding Fields: A theory of learning with physiological implications. Journal of Mathematical Psychology, 1969, p. 209-239.

[16] Banon, G. J. F.; Barrera J. Minimal representations for translation-invariant set mappings by morphology mathematical. SIAM Journal Applied Mathematic, 51(6):1782-1798, Dec. 1991.

[17] Kohonen, T. Self-organization and associative memory $\left(3^{\text {rd }}\right.$ ed. $)$, Berlin: Springer-Verlag, 1989.

[18] Banon, G. J. F.; Barrera J. Decomposition of mappings between complete lattices by mathematical morphology
- part I: general lattices. Signal Processing, 30(3):299327, Feb. 1993.

[19] Maragos, P. A represetation theory for morphological image and signal processing, IEEE-Transactions on Pattern Analysis and Machine Intelligence, vol. II. No. 6, June, 1989.

[20] Banon, G. J. F. Morphological decomposition of image operators for extreme gray-level computation and localization. Brazilian Workshop'97 on Mathematical Morphology. São José dos Campos, Instituto Nacional de Pesquisas Espaciais, número INPE-7075-PRE/3013, 1997. Repositório da URLIb: <dpi.inpe.br/banon/1997/01.30.14.02>.

[21] Faria, S. Uma Abordagem Morfológica para Casamento de Padrões. São José dos Campos. Dissertação (Mestrado em Computação Aplicada) - Instituto Nacional de Pesquisas Espaciais, número INPE-6346TDI/597, 1997. Repositório da URLib: <dpi.inpe.br/faria/1997/04.16.15.30>.

[22] Silva, F.A.T.F. Rede morfológica não supervisionadaRMNS. Doutorado em computação aplicada, INPE-São José dos Campos, 1998.

[23] Heijmans, H. J. A. M. Theoretical aspects of gray-level morphology. IEEE Transactions on Pattern Analysis and Machine Intelligence, v. 13, 1991, p. 568-582.

[24] Serra, J. Thickenings, thinnings. Centre de morphologie mathématuque, école nationale supérieure des mines de Paris, june, 1987.

[25] Banon, G. J. F.; Faria, S. D. Morphological approach for template matching. SIBGRAPI'97, IEEE Computer Society, 1997, p. 171-178, número INPE-7110PRE/3048. Repositório da URLib: <dpi.inpe.br/banon/1997/05.23.15.08>.

[26] Serra, J. Image Analysis and mathematical Morphology. Volume 1. London: Academic Press, 1982.

[27] Serra, J. Image Analysis and mathematical Morphology. Volume 2: Theoretical Advances. London: Academic Press, 1988. 